\title{
Gastrojejuno-Colic Fistula: Case Report
}

\author{
Momcilo Stosic $^{1 *}$, Marko Gmijovic ${ }^{2}$, Igor Stojanovic ${ }^{1}$ and Kosta Zdravkovićc ${ }^{3}$ \\ ${ }^{1}$ Department of surgery, Serbia \\ ${ }^{2}$ Clinic for general surgery, Serbia \\ ${ }^{3}$ Daily oncology hospital, Serbia
}

*Corresponding author: Momcilo Stosic, Department of surgery, Serbia

Submission: 侮February 05, 2019; Published: 眥February 13, 2019

\begin{abstract}
Introduction: Since the beginning of the 1990s, surgical procedures for peptic ulcer disease have been very rare as a result of administering medical treatments such as proton pump inhibitors and anti-Helicobacter Pylori therapy. The post- surgical complications may be noticed presently, i.e. 10, 20 or more years after the initial surgical treatment. Gastrojejunocolic fistula (GJCF) is one of the complications. The symptoms include chronic diarrhea and weight loss. Ingested food passes through the fistula, bypassing all of the small intestine and a part of the colon. Contrast examination is the most sensitive diagnostic tool. The treatment is surgical, preceded by suitable protein-electrolyte preparation. Unlike previous years, surgical approach is now a single-stage procedure.
\end{abstract}

Case report: A 60- year old man was admitted to hospital, complaining of progressive weight loss, chronic diarrhoea, and feculent breath. The clinical examination took a few months, including rare disease diagnostics (APUD tumours). Endoscopic examinations were performed repeatedly, but none showed a minor fistula, as it had been at the beginning. The diagnosis was made by contrast examination of the gastro duodenum. After electrolyte imbalance and nutritional deficiencies were resuscitated, a re-resection of the stomach, anastomotic ulcer, proximal jejunum and transverse colon was performed in a single-stage procedure. The reconstruction of the gastro-jejunum was performed by Roux- en-Y technique, and colocolic anastomosis was performed during a single-stage procedure. The patient was discharged from the hospital on the 11th postoperative day, but he continued treatment for R-y stasis syndrome. The weight gain after 6 months was $15 \mathrm{~kg}$, and the patient did not report diarrhea or feculent breath.

Conclusion: The modern diagnostic methods might have unjustly challenged the importance of contrast examination of the digestive tract. In our case, contrast radiography was used to make a diagnosis. Chronic diarrhea symptom is present in infectious enterocolitis, while Crohn's disease or a malignancy may also be suspected. In case when more frequent diseases are excluded, the mentioned fistula should be considered. Nowadays, GJCF surgery is performed as a single-stage procedure after providing adequate protein-electrolyte preparation.

Keywords: Dermatofibrosarcoma protuberans, Mohs surgery, Vulva

\section{Introduction}

Gastrojejunocolic fistula (GJCF) is the second non-malignant surgical cause of chronic diarrhea. The incidence rate is low. Soybel et al. [1] listed 108 published studies. If in the 1960s reports on 30 cases were not uncommon, in the last 20 years reports of no more than 5 cases were published, Puia et al. [2] quoted. GJCF may be caused by peptic ulcer disease, before or after stomach resection. It develops more frequently after the resection as a result of stomal ulcer. Incomplete resection or inadequate vagotomy might be the causes of the condition, or hypersecretion may be found in a patient.

Since the 1990s, the number of stomach resection and other surgical procedures for peptic ulcer disease has been decreasing due to administering medical therapy such as proton pump inhibitors and regiments for Helicobacter pylori eradication. Presently, surgery is rarely performed. The aim of the study is to highlight the crucial symptom of non-malignant cause of GJCF-chronic diarrhoea, as well as the possibility of the fistula developing a decade or more after the initial surgery.

\section{A Case 60-year port}

Old man was admitted to surgical department for the first time for clinical examination two years ago, after having been examined by other medical specialists. He mainly complained of occasional abdominal pain, chronic diarrhoea and minor weight loss. Ten years prior, the patient had undergone resection of the stomach for peptic ulcer disease: Billroth II with R-y gastro-jejuno-anastomosis. The gallbladder was removed at the same time. The patient was presented with post-operative ventral hernia. He consumed 3 to 4 shot glasses of fruit brandy on a day-to-day basis. Laboratory tests were done several times. The first laboratory findings were within normal range and with no signs of malnutrition. Colonoscopy was negative, gastroscopy showed gastric erosion, while abdominal 
ultrasound findings were normal. The patient was discharged from the hospital, being diagnosed with "enterocolitis" and received anti-ulcer and anti-enteritis therapy.

During the following year, the patient complained of persistent watery diarrhoea (3-4 times a day) and further weight loss, and thus he was referred three times to two tertiary institutions with suspicion of APUD tumour. Laboratory tests were performed several times, endoscopy of the upper GI tract was done two times, gastrojejunal examination was performed once by capsule endoscopy, and a colonoscopy was done. The diagnosis was not established. Two years after presenting with the same symptoms (diarrhoea, severe weight loss-more than $20 \mathrm{~kg}$ ), the patient was admitted for treatment. The new symptom was a feculent breath. An X-ray contrast examination was done (gastrografin enema and barium meal) and R-y gastrojejunoanastomotic-colic fistula was confirmed (Figure 1).

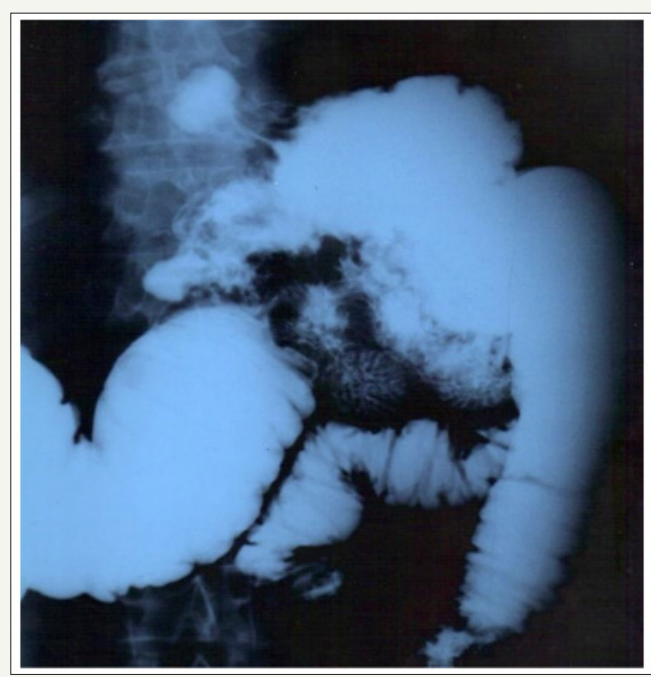

Figure 1: Gastrojejunocolic fistula.

After restoring protein, albumin and electrolyte balance, the patient underwent surgery. The resection of gastrojejunal anastomosis with ulcer and a partially affected " $\mathrm{T}$ " transverse colon was performed. Reconstruction was done at the same time. Recovery was uneventful. The patient was discharged on the 12 th day after the surgery. After discharge, the patient presented with postprandial vomiting and upper abdominal distension, so followup gastroscopy and contrast examination were performed, and it was confirmed that the transit was regular. The stool was normal, there was no feculent breath. The patient was treated conservatively for anastomosis and R-y stasis syndrome. After 9-month follow-up, the patient remained well, with $15 \mathrm{~kg}$ weight gain, and all laboratory tests and abdominal US examination were within normal range.

\section{Discussion}

Classic triad of symptoms related to GJCF includes weight loss, chronic diarrhoea and feculent breath. Pain is occasional and uncharacteristic. The patient was presented with the abovementioned symptoms. In the present, the diagnosis of the complication is usually delayed. The reason for this is the time of the incidence, approximately 15-20 years after the original surgery, most frequently after Billroth II resection $[3,4]$. The most important diagnostic tools are endoscopy and contrast examination [5]. CT scan is used to assess the condition of nearby structures after diagnosis has been made. Contrast examination is more reliable than endoscopy (90-100\%) [6,7]. Recently, endoscopy and colonoscopy have been recommended for the diagnosis of GJFC fistula to exclude other gastrointestinal diseases [8]. Kumar G et al. [9] state:
Gastroscopy and colonoscopy are not first line examinations for the gastrocolic fistula diagnosis, since the communication can be easily missed between the gastric folds or the colonic haustra, unless the fistulous orifice is big enough and can be visualized [9,10]. Our case study also shows that diagnosis was made by means of contrast examination, although endoscopy was repeated, including capsule endoscopy.

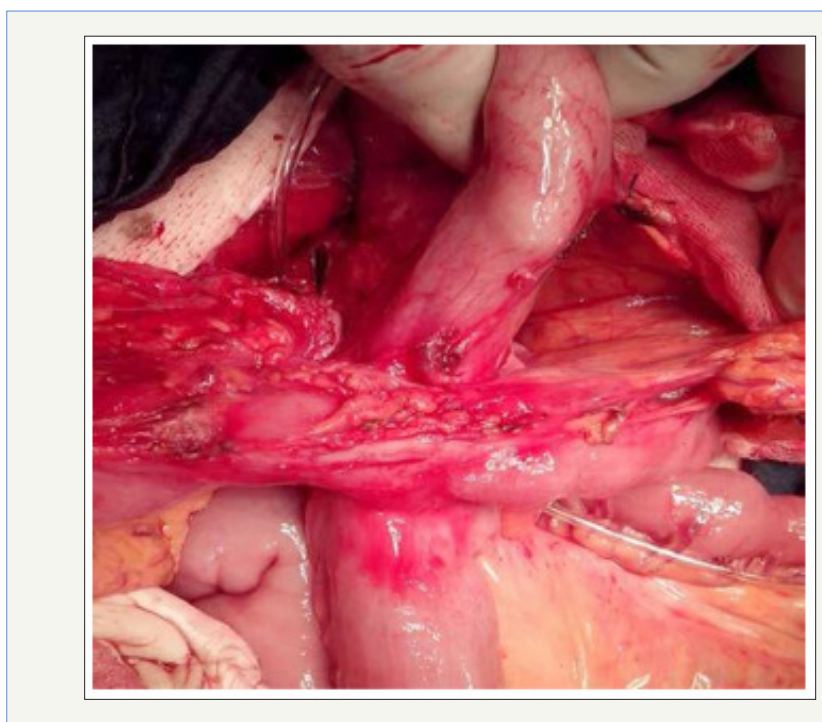

Figure 2: GJCF retrocolica, omentum removed and adhesions.

Since malnutrition is present with the patients, adequate preoperative care is of great importance (albumin, protein, FE). In 
1940-60s, a simple colostomy was recommended. Then followed the age of two or three-stage surgery [5]. This surgical strategy reduced mortality by $40 \%$. The development of parenteral nutrition led to open single-stage surgery with primary reconstruction. It meant that a radical en-bloc resection was performed involving a re-resection gastrectomy, partial transverse colon and jejunum resection; restoration of bowel continuity was applied by gastroenterostomy and colocolostomy [10]. The presented case shows the mentioned treatment (Figure 2-5). The latest management of GJCF in a singlestage laparoscopic procedure has been proving its validity $[11,12]$.

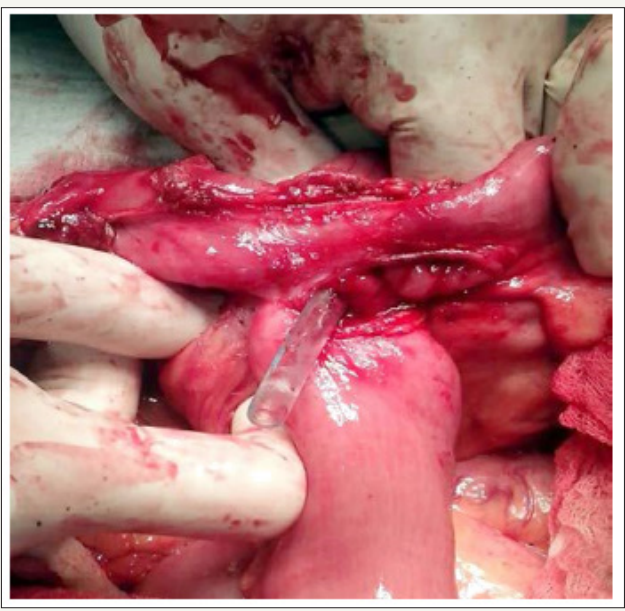

Figure 3: GJCF and nasogastric probe.

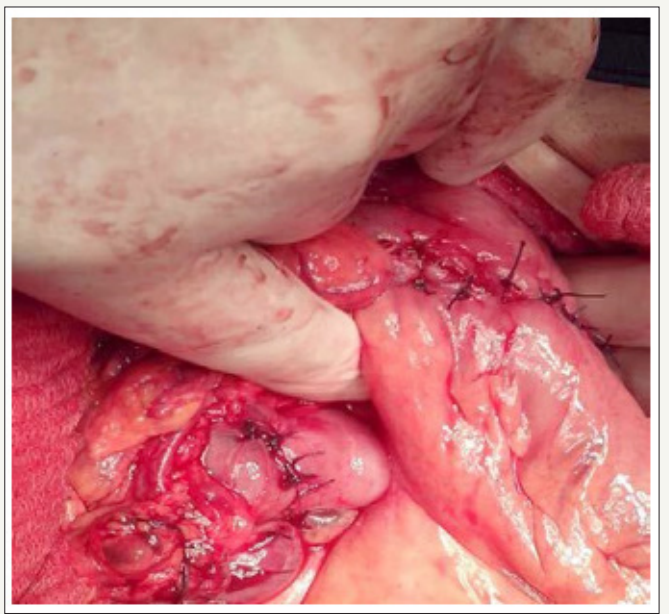

Figure 4: GJ anastomosis antecolica and colo-colo anastomosis.

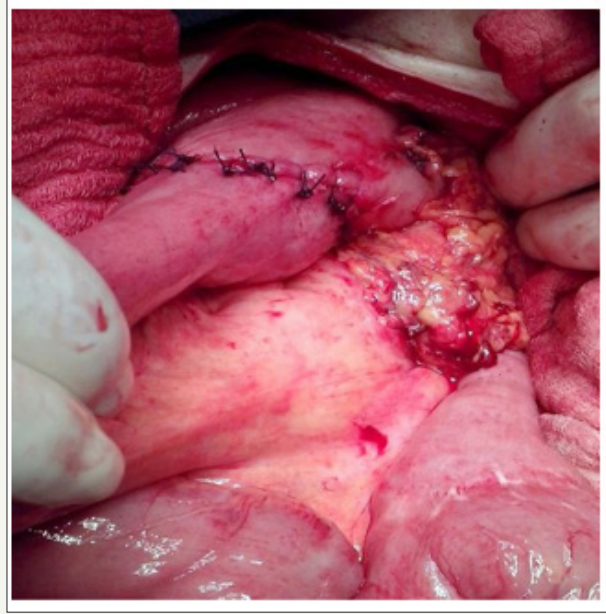

Figure 5: GJ-anastomosis high above colonic anastomosis. 


\section{Conclusion}

The case describes a rare disease since the number of surgeries for peptic ulcer disease has exceptionally decreased. Therefore, the diagnosis is delayed. GJCF results from gastric resection of the stomach, performed 10 years prior. Despite the contemporary methods of diagnostics, contrast examination was crucial and most effective. An adequate parenteral pre-operative care is of great importance in preventing leakage. Surgical treatment of choice is en-bloc resection.

\section{References}

1. Soybel DI, Kestenberg A, Brunt EM, Becker JM (1989) Gastrocolic fistula as a complication of benign gastric ulcer: Report of four cases and update of the literature. British Journal of Surgery 76(12): 1298-1300.

2. Puia IC, Iancu C, Bãlã O, Munteanu D, Al-Hajjar N, et al. (2012) Gastrojejunocolic fistula: report of six cases and review of the literature. Chirurgia 107: 52-54.

3. Kim KH, Jee YS (2013) Gastrojejuno-colic fistula after gastrojejunostomy. J Korean Surg Soc 84(4): 252-255.

4. Desta GK, Gebremeskel WW, Wasihun AG (2015) Gastrojejunocolic fistula after gastrojejunostomy in Ayder referral hospital Northern Ethiopia: A report of two cases. Ann Med Surg (Lond) 4(4): 448-451.
5. Kece C, Dalgic T, Nadir I, Baydar B, Nessar G, et al. (2010) Current diagnosis and management of gastrojejunocolic fistula. Case Rep Gastroenterol 4(2): 173-177.

6. Ohta M, Konno H, Tanaka T, Baba M, Kamiya K, et al. (2002) Gastrojejunocolic fistula after gastrectomy with Billroth II reconstruction: report of a case. Surg Today 32(4): 367-370.

7. Thoeny RH, Hodgson JR, Scudamore HH (1960) The roentgenologic diagnosis of gastrocolic and gastrohejunocolic fistulas. Am J Roentgenol Radium Ther Nucl Med 83: 876-881.

8. Nussinson E, Samara M, Abud H (1987) Gastrojejunocolic fistula diagnosed by simultaneous gastroscopy and colonoscopy. Gastrointest Endosc 33(5): 398-399.

9. Kumar G, Razzaque M, Naidu V, Barbour E (1976) Gastrocolic fistulae in benign peptic ulcer disease. Ann Surg 184(2): 236-240.

10. Stamatakos M, Karaiskos I, Pateras I, Alexiou I, Stefanaki C, et al. (2012) Gastrocolic fistulae; From haller till nowadays. Int J Surg 10(3): 129-133.

11. Chung DP, Li RS, Leong HT (2001) Diagnosis and current management of gastrojejunocolic fistula. Hong Kong Med J 7(4): 439-441.

12. Takemura M, Hamano G, Nishioka T, Takii M, Mayumi K, et al. (2011) One-stage laparoscopic-assisted resection of gastrojejunocolic fistula after gastrojejunostomy for duodenal ulcer: a case report. J Med Case Rep 5: 543.

\section{Advancements in Case Studies}

\section{Benefits of Publishing with us}

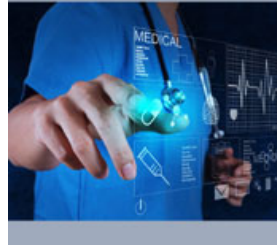

- High-level peer review and editorial services

- Freely accessible online immediately upon publication

- Authors retain the copyright to their work

- Licensing it under a Creative Commons license

- Visibility through different online platforms 